\title{
Challenges in Addressing Community Concerns Regarding Clusters of Multiple Sclerosis and Potential Environmental Exposures
}

\author{
Dhelia M. Williamsona Judy P. Henry ${ }^{b}$ \\ aDivision of Health Studies, Agency for Toxic Substances and Disease Registry, Atlanta, Ga. and \\ bBureau of Epidemiology and Toxicology, Texas Department of Health, Austin, Tex., USA
}

\section{Key Words}

Multiple sclerosis · Cluster investigations •

Environmental exposure

\begin{abstract}
Citizens living around hazardous waste sites in the USA have expressed concern to public health officials at the local, state and federal level about a perceived high prevalence of multiple sclerosis (MS) in their communities. Many believe the occurrence of the disease is directly linked to exposure to chemical agents from the nearby hazardous waste site. Although the public's concern regarding these clusters should be addressed, epidemiologists have long known that evaluating perceived clusters is rarely fruitful for identifying an etiologic agent. In order to adequately address concerns regarding clusters of MS, as well as examining the role of environmental exposures and genetic susceptibility in the causal mechanism of disease, several activities need to be conducted including characterizing the occurrence of disease, developing a standardized case definition and establishing partnerships to develop innovative research techniques. Only with collaboration across disciplines and lessons learned from past research will we be able to effectively guide research efforts directed at determining the etiology of this disease.
\end{abstract}

Copyright $\odot 2004$ S. Karger AG, Basel

\section{Introduction}

Multiple sclerosis (MS) is a demyelinating disease of the central nervous system and one of the most common causes of neurological disability in young adults [1]. The diagnosis of MS is unusual before adolescence, rises steadily from the teens to age 35 , and then declines gradually thereafter [2,3]. Approximately twice as many women are affected by MS as men and Caucasians of northern and central European ancestry are at highest risk of developing MS [3-6]. The progression of the disease is variable with the clinical course of MS varying from an asymptomatic condition to an aggressive form that can rapidly lead to severe disability $[3,7]$. Most commonly, the clinical course involves a series of remissions and relapses that may become progressively more severe over time. MS usually is not a fatal disease, but it often results in decreased quality of life.

People living around hazardous waste sites in the USA have expressed concern to local, state and federal public health officials about perceived increases in the prevalence of MS in their communities. Many believe the occurrence of the disease is directly linked to exposure to chemical agents from the hazardous waste site [8-10]. The Agency for Toxic Substances and Disease Registry (ATSDR), a US federal public health agency, and collaborators at local and state health agencies, work to prevent

Dhelia M. Williamson

Division of Health Studies, Agency for Toxic Substances and Disease Registry 1600 Clifton Road MS E-31

Atlanta, GA 30333 (USA)

Tel. +1 404498 0586, Fax +1 404498 0079, E-Mail djw8@cdc.gov 
exposure and adverse health effects associated with exposure to hazardous substances from waste sites, unplanned releases and other sources of pollution present in the environment. In more and more communities where these agencies work, they are being asked to investigate purported clusters of MS, amyotrophic lateral sclerosis, lupus or other neurologic diseases. However, even the most basic tools with which to conduct these types of investigation are lacking including background prevalence comparison estimates, an understanding of biologically plausible mechanisms for contributions of environmental contaminants to the disease process, and knowledge of causal disease mechanisms.

The US Centers for Disease Control and Prevention (CDC) developed guidelines for investigating disease clusters that have been used successfully in the investigation of purported cancer and birth defect clusters [11]. One of the preliminary steps, as outlined in the CDC guidelines, is to determine if there is an actual excess of the disease of concern in the community reporting the cluster. When a community identifies an apparent cluster of MS, however, it is difficult to address these concerns because the data needed to estimate MS prevalence cannot be obtained. MS is not a reportable disease and national prevalence data are neither current nor readily available for comparison. The inability of public health agencies to address the most basic question of whether there is an excess of MS in the community results in frustration for the community and loss of credibility for public health officials.

This article will provide an overview of (1) challenges faced by local, state and federal public health agencies in the USA when asked to examine clusters of MS, and (2) the role potential environmental factors may play in the development of MS. Although the focus of this paper is MS, many of the issues discussed pertain to other noninfectious diseases as well. Recommendations for future public health activities are also discussed.

\section{Challenges}

There are several challenges public health officials face when investigating apparent clusters of MS in communities. These challenges include a lack of available data on MS, inability of cluster studies to address community concerns, causal agents have not been identified, limitations of environmental epidemiology and inadequate infrastructure of public health agencies.

\section{Lack of Available Data on MS}

According to CDC guidelines for investigating clusters, the first step is to determine if there is an excess of disease in the community by comparing the number of reported cases to an 'expected' number of cases based on a reference population [10]. The expected number of cases is often derived from an existing disease registry, such as those for cancer or birth defects. Ideally, the registry information would be specific to the state or region in which the cluster was reported and be timely; however, there are no population-based registries or ongoing surveillance efforts for MS in the USA. Therefore, the (expected) number of MS cases has been established using prevalence estimates from published articles. These estimates, however, are not current and vary widely due to the methodology and population sample used.

The National Health Interview Survey (NHIS) is a health survey conducted annually by the CDC that collects data on self-reported illnesses. Using NHIS data collected during 1989-1994 the estimated prevalence of MS is 87 cases per 100,000 population in the civilian noninstitutionalized population [11]. In 1975, the National Institute of Neurological Disorders and Stroke (NINDS) conducted a survey of physicians and hospitals by using a probability sample of healthcare providers considered most likely to treat MS patients in the contiguous 50 states and reported a prevalence of 57.8 cases of MS per 100,000 population [12]. The prevalence of MS for specific locales has been reported since 1970 with a prevalence range from 22 to 160 per 100,000 population. The highest prevalence for a specific geographic area was reported in a 1985 Minnesota study that identified cases of MS in Olmsted County from 1905 through 1984 by using the centralized diagnostic index at the Mayo Clinic [13]. The diagnostic index allowed for the identification and retrieval of medical charts for patients that met study eligibility and who had been diagnosed with MS by a clinical neurologist. This and other evidence suggests there are regional differences in the prevalence of MS in the USA, which makes using national estimates to establish an 'expected' number of cases even more problematic $[4,11,12,14$ 18].

An accurate estimate of the underlying disease burden in a population is required to appropriately evaluate a possible excess in a timely, efficacious and credible manner. This is illustrated by a recently completed MS cluster investigation in El Paso, Texas. Depending on the reference population used in this investigation, the standardized morbidity ratios ranged from 1.05 (95\% confidence interval (CI) 0.57-1.76) using the Olmsted County data, 
1.93 (95\% CI 1.06-3.24) using the NINDS National Survey, and 2.80 (95\% CI 1.52-4.66) using the NHIS National Survey [19]. At the extremes, these estimates based on national published data could be interpreted as there being no excess of disease or as there being an approximate threefold excess of MS in the study population. In 1999, ATSDR funded three states to conduct a pilot MS surveillance project. Based on preliminary data from the Texas pilot surveillance project, a re-analysis of the El Paso MS cluster using the Texas-specific data indicates the standardized morbidity ratio may approach 8.0. Citizens require and deserve a less ambiguous answer to their questions concerning an excess of disease in their community. Public health agencies also need less ambiguous information when making a decision as to whether additional resources should be committed to pursuing an etiologic investigation.

\section{Inability of Cluster Studies to Address Community Concerns}

Although public health officials understand that investigating disease clusters is usually not fruitful for identifying an etiologic agent, they are regarded as unresponsive and uncaring by community members if they do not pursue additional studies once a cluster has been confirmed. If further investigations are conducted and the results are inconclusive, public health officials are sometimes regarded with mistrust. Overall, cluster investigations establish expectations that typically cannot be met by an epidemiologic investigation.

If an excess of disease is identified in a community, residents generally request that public health agencies conduct additional studies to determine the specific cause of the cluster. However, previous studies of cluster investigations of MS in the USA have shown that the probability of finding a discernable cause is low. There are three main reasons for this. First, all individuals included in reported clusters may not meet a uniform case definition for MS based on a set of generally accepted criteria such as the Poser or McDonald criteria [20, 21]. In a cluster investigation in Los Alamos County, New Mexico, 26 of 53 potential cases met the diagnostic criteria of MS after review of medical records [8]. Second, relevant exposures of interest are unmeasured, particularly historical exposures that are needed to help determine the latency of disease. Cluster investigations of MS in the USA have typically been conducted in areas where there is local concern regarding exposure from a hazardous waste site [7-9]. Unfortunately, past exposure information at the individual or pertinent geographic area level is generally not avail- able so studies rely on self-reported exposure information. However, even when occupational clusters are investigated, it is difficult to identify causal agents [22]. Third, the small number of cases in such (clusters) often limits the ability to differentiate between an excess of etiologic interest and a chance occurrence. MS cluster investigations have been conducted with less than 10 individuals $[23,24]$ although most have less than 40 individuals [7-9].

\section{Causal Agents Have Not Been Identified}

Although the cause of MS is unknown, evidence indicates that it is a complex disease with a multifactorial etiology determined by both environmental factors and genetic susceptibility [4, 25-38]. A number of environmental exposures - heavy metals, infectious agents, solvents, diet and smoking - have been investigated as potential risk factors for disease but no consistent associations have been established [25, 29, 30]. It is currently hypothesized that one or more environmental exposures in a genetically susceptible individual may trigger the development of disease [4, 25-27].

Studies focusing on the genetic component of MS have focused on the apparent immune mechanism of the disease including the major histocompatibility complex, particularly the human leukocyte antigen region, immunoglobulin heavy chain, T-cell receptor, tumor necrosis factor and myelin basic protein loci [3, 31]. Association, classic linkage and affected sib-pair linkage studies have been conducted examining the role these factors play in the onset of disease, but the results have been conflicting. With the exception of the human leukocyte antigen genes, there have been no consistently replicated findings of linkage to any candidate genes [28, 32-34]. These results suggest that a single locus model for MS is unlikely.

Several viruses are thought to be associated with MS including canine distemper, measles, herpes (HHV-6), rubella (German measles), human T-cell lymphotrophic virus 1 and Epstein-Barr although subsequent studies have not substantiated all of these reports [35-37]. Because the relapsing-remitting phase of MS in many ways is analogous to the recurrence of herpes virus infections, a virus from this group has been an attractive etiologic candidate [39]. Studies also suggest that the critical time period for environmental exposures is prior to puberty [3, 4, 17]. Lack of an identified causal agent(s) - either environmental or genetic - makes studying this disease difficult.

Neuroepidemiology 2004;23:211-216 


\section{Limitations of Environmental Epidemiology}

The goal of environmental epidemiology is to evaluate whether physical, biologic and chemical factors in the external environment (i.e., in air, soil and water) are associated with the occurrence of disease in a population. Characterizing exposure is an important limitation of these studies. People are exposed to many different toxic pollutants throughout their lives and the health impact of these complex mixtures is unknown. In addition, evaluating the effect of environmental exposures is difficult unless the exposure of concern is persistent in the environment or can be reconstructed from existing records. When investigating a disease such as MS, which has a long and variable latency period, this process is even more complex because it is difficult to characterize historical exposures to individuals during the time period of concern. Another problem with conducting epidemiologic research of environmental exposures is accounting for recall bias. People with MS may have better recall of potential exposures than people without the disease. Also, the publicity associated with cluster investigations can make it difficult to obtain unbiased responses in interviews and on questionnaires.

\section{Inadequate Infrastructure of Public Health Agencies}

Public health agencies need and want to respond to community concerns about potential disease clusters. Although most health departments have developed protocols to respond to calls about clusters of cancer or birth defects, local, state and federal health agencies lack programs focusing specifically on MS. Even if the CDC guidelines for investigating clusters are used, public health officials are not able to determine if there is an excess of MS in the community because a geographic-specific and timely reference population is not available to calculate the number 'expected'. Due to the lack of incidence or prevalence data for MS, when a call is received about a perceived cluster of MS in a community, public health agencies appear to be unprepared or unresponsive. For example, a concerned resident with MS living in Ohio first contacted the local health department in 1984 to report that there were 27 individuals with MS that had lived in the village (population 4,500). Although the local health department lacked resources to conduct a full epidemiological study, they were able to confirm an MS diagnosis for the majority of the 27 individuals. It was not until ATSDR was able to provide funding to the local health department that a more comprehensive investigation was initiated. Their results from this investigation will be used to determine if there is an excess of MS in the village compared to the county. However, this study will not be able to address etiologic issues. As is illustrated in the Ohio example, community residents become very frustrated when their concerns are not investigated in a timely manner. Public health officials became frustrated because they have neither the staff nor the resources to address the issue.

\section{Recommendations}

Epidemiologists have long known that evaluating perceived clusters is rarely fruitful for identifying an etiologic agent, however the public's concern regarding these clusters should be addressed. In order to adequately address questions regarding the causes of MS and the potential role of environmental exposures, several activities need to be undertaken: surveillance techniques need to be developed in order to characterize the occurrence of MS in the USA, a standardized case definition needs to be used for these surveillance activities, and innovative research techniques need to be encouraged among MS researchers in various fields.

\section{Characterize the Occurrence of Disease}

The number of individuals in the USA who are affected by MS and the resultant magnitude of the impact of the disease on the public's health are unknown. In order to understand the clinical, public health and economic importance of MS, it is necessary to have accurate estimates of the incidence and prevalence of MS at the national, state and local level [40]. Surveillance techniques need to be developed to so that accurate incidence and prevalence data will be available to public health agencies. Access to this information will allow questions from concerned communities about the occurrence of MS in their area to be answered in a timely manner. Further, the information available from a national surveillance system will serve to guide research efforts when conducting studies to identify potential causes of this disease, including the possible contribution of environmental contaminants.

\section{Use of a Standardized Case Definition}

The diagnosis of MS is often difficult because there is no single clinical sign or diagnostic test that is unique to this disease and because there is wide variation in presentation of signs and symptoms. In order to have an effective surveillance system, a clear case definition is needed. Currently, two diagnostic criteria are being used by neu- 
rologists: the Poser criteria [20] and the McDonald criteria, which differ in important ways [21]. Unlike the Poser criteria, the McDonald criteria integrate magnetic resonance imaging (MRI) into the overall diagnostic scheme and include the diagnosis of primary progressive disease. For surveillance purposes, either criteria may be acceptable for determining incidence and prevalence of MS.

\section{Establish Partnerships to Develop Innovative Research Techniques}

Previous studies support both genetic and environmental components of susceptibility to MS. In the USA, many studies currently underway are not examining both components concurrently and collaboration among researchers with different areas of expertise is minimal. Future research should examine the interaction between genetic and environmental factors involved in the etiology and progression of MS. In addition, the USA should follow the examples of Europe and Canada and establish partnerships between researchers with expertise in genetics, neurology, immunology, epidemiology and toxicology.

\section{Discussion}

Although MS causes significant chronic morbidity and disability and affects primarily young and middle-aged adults, it has not received much attention from public health agencies. In order to address this deficiency, the ATSDR is completing a cooperative agreement to determine the prevalence of MS in three states - Ohio, Missouri and Texas. The primary data source for MS case ascertainment is medical records from neurologists' offices which are reviewed and verified by each study area's consulting neurologist. Cases status was determined by applying both the Poser and McDonald criteria. The information gained from this project will help fill a data gap and will assist in answering questions about the prevalence of MS in concerned communities in these states. In addition, the methods developed during this project are serving as a prototype for other surveillance activities supported by ATSDR including additional studies to determine the prevalence of MS and amyotrophic lateral sclerosis in five different geographic areas where there are community concerns regarding an increased prevalence of these diseases.

On March 21, 2002, new legislation was introduced in both the US Senate and House of Representatives that would establish a Nationwide Health Tracking Network to track chronic diseases. The network will collect information regarding when and where chronic diseases occur, as well as information about potentially related environmental factors. In addition, CDC announced the availability of funds for a cooperative agreement program in 2002 to support development of a National Environmental Public Health Tracking (Surveillance) Program. The purpose of this CDC program is to (1) develop the components of a standards-based, coordinated and integrated environmental public health tracking (surveillance) system at state and national levels that will allow linking of health effects data with human exposure and environmental hazards data, and (2) increase environmental public health capacity at the local, state and national levels. National programs such as these will help address questions regarding trends in the prevalence of chronic diseases such as MS and will assist in identifying potential areas of study with regard to suspected environmental exposures.

To examine the causal mechanisms of MS, including the role of environmental exposures and genetic susceptibility, collaboration among researchers with varied expertise is essential. Currently, ATSDR is collaborating with local, state and federal public health officials, neurologists, geneticists, other researchers and individuals affected with MS to guide research efforts directed at potential etiologies of this disease. Past research, including results from cluster investigations, has demonstrated that large-scale studies are needed to clarify potential causes of this disease. ATSDR is funding a national multi-site casecontrol study to examine the role of environmental factors, infectious disease and genetic susceptibility in the development of MS. In addition to evaluating potential causes of this disease, information from this type of study could guide planning of effective prevention programs and interventions to help reduce the burden of this disease in future generations. 


\section{References}

1 Joy JE, Johnson $\mathrm{J} \mathrm{Jr}$ (eds): Institute of Medicine - Multiple Sclerosis: Current Status and Strategies for the Future. Washington, National Academy Press, 2001. Available from URL: http://books.nap.edu/books/0309072859/html

2 Hauser S: Multiple sclerosis and other demyelinating diseases; in Isselbacher KJ, Martin JB (eds): Harrison's Principles of Internal Medicine. New York, McGraw-Hill, 1994, pp 2289_ 2295.

3 Sadovnick AD, Dyment D, Ebers GC: Genetic epidemiology of multiple sclerosis. Epidemiol Rev 1997;19:99-106.

4 Hogancamp WE, Rodriguez M, Weinshenker BG: The epidemiology of multiple sclerosis. Mayo Clin Proc 1997;72:871-878.

5 Kesselring J: Multiple Sclerosis. Cambridge/ UK, Cambridge University Press, 1997.

6 Schwartz RS: Autoimmunity and autoimmune diseases; in Paul WE (ed): Fundamental Immunology. New York, Raven Press, 1993, pp 1079-1080

7 Helmick CG, Wrigley M, Zack MM, Bigler WJ, Lehman JI, Janssen RS, Hartwig CE, Witte JJ Multiple sclerosis in Key West, Florida. Am J Epidemiol 1989;130:935-949.

8 Hoffman RE, Zack MM, Davis LE, Burchfiel $\mathrm{CM}$ : Increased incidence and prevalence of multiple sclerosis in Los Alamos County, New Mexico. Neurology 1981;31:1489-1492.

9 Hopkins RS, Indian RW, Pinnow E, Conomy $\mathrm{J}$ : Multiple sclerosis in Galion, Ohio: Prevalence and results of a case-control study. Neuroepidemiology 1991;10:192-199.

10 Centers for Disease Control and Prevention: Guidelines for investigating clusters of health events. MMWR 1990;39(RR-11).

11 Noonan CW, Kathman SJ, White MC: Prevalence estimates for MS in the United States and evidence of an increasing trend for women. Neurology 2002;58:136-138.

12 Baum HM, Rothschild BB: The incidence and prevalence of reported multiple sclerosis. Ann Neurol 1981;10:420-428.

13 Wynn DR, Rodriguez M, O'Fallen M, Kurland LT: A reappraisal of the epidemiology of multiple sclerosis in Olmsted County, Minnesota. Neurology 1990;40:780-786.
14 Kurtzke JF: Multiple sclerosis: Changing times. Neuroepidemiology 1991;10:1-8.

15 Kurtzke JF: Geography in multiple sclerosis. J Neurol 1977;215:1-26.

16 Hernan MA, Olek MJ, Ascherio AJ: Geographic variation of multiple sclerosis incidence in two prospective studies of US women. Neurology 1999;53:1711-1718.

17 Poser CM: The epidemiology of multiple sclerosis: A general overview. Ann Neurol 1994; 36(suppl 2):180-193.

18 Wadia NH, Bhatia K: Multiple sclerosis prevalence in the Zoroastrians (Parsis) of India. Ann Neurol 1990;28:177-179.

19 Agency for Toxic Substances and Disease Registry: El Paso multiple sclerosis cluster investigation, El Paso, El Paso County, Texas. Atlanta, US Department of Health and Human Services, 2002.

20 Poser CM, Paty DW, Scheinberg LC, McDonald WI, Davis FA, Ebers GC et al: New diagnostic criteria for multiple sclerosis: Guidelines for research protocols. Ann Neurol 1983;13: 227-231.

21 McDonald WI, Compston A, Edan G, Goodkin D, Hartung HP, Lublin FD et al: Recommended diagnostic criteria for multiple sclerosis: Guidelines from the international panel on the diagnosis of multiple sclerosis. Ann Neurol 2001;50:121-127.

22 Stein EC, Schiffer RB, Hall WJ, Young N: Multiple sclerosis and the workplace: Report of an industry-based cluster. Neurology 1987;37: 1672-1677.

23 Eastman R, Sheridan J, Poskanzer DC: Multiple sclerosis clustering in a small Massachusetts community, with possible common exposure 23 years before onset. N Engl J Med 1973;209: 793-794.

24 Koch MJ, Reed D, Stern R, Brody JA: Multiple sclerosis: A cluster in a small northwestern United States community. JAMA 1974;228: 1555-1557.

25 Kahana E: Epidemiologic studies of multiple sclerosis: A review. Biomed Phamacother 2000;54:100-102.

26 Marrie RA, Wolfson SC, Sturkenboom MC, Gout O, Heinzlef O, Roullet E, Abenhaim L: Multiple sclerosis and antecedent infections. Neurology 2000;54:2307-2310.
27 Noseworthy JH, Lucchinetti C, Rodriguez M, Weinshenker BG: Multiple sclerosis. N Eng J Med 2000;343:938-952.

28 Willer CJ, Ebers GC: Susceptibility to multiple sclerosis: Interplay between genes and environment. Curr Opin Neurol 2000;13:241-247.

29 Minden SL, Marker WD, Harrold LN, Dor A Multiple sclerosis: A statistical portrait. National Multiple Sclerosis Society, 1993.

30 Weinshenker BG: Epidemiology of multiple sclerosis. Neuroepidemiology 1996;14:291308.

31 The Multiple Sclerosis Genetics Group: A complex genomic screen for multiple sclerosis underscores a role for the major histocompatibility complex. Nat Genet 1996;13:241-247.

32 Compston A: The genetics of multiple sclerosis. J Neurovirol 2000;6:S5-S9.

33 Dyment DA, Sadnovick AD, Ebers GC: Genetics of multiple sclerosis. Hum Mol Genet 1997; 6:1693-1698

34 Noseworthy JH: Progress in determining the causes and treatment of multiple sclerosis. Nature 1999;399:A40-A47.

35 Casetta I, Granieri E: Clinical infections and multiple sclerosis: Contribution from analytical epidemiology. J Neurovirol 2000;6(suppl 2):147-151.

36 Hernan MA, Zhang M, Lipworth L, Okel MJ, Ascherio A: Multiple sclerosis and age at infection with common viruses. Epidemiology 2001;12:301-306.

37 Ascherio A, Zhang SM, Hernan MA, Olek MJ Coplan PM, Brodovicz K, Walker AM: Hepatitis $B$ vaccination and the risk of multiple sclerosis. N Engl J Med 2001;344:327-332.

38 Ascherio A, Munch M: Epstein-Barr virus and multiple sclerosis. Epidemiology 1999;11:220 223.

39 Haahr S, Munch M: The association between multiple sclerosis and infection with EpsteinBarr virus and retrovirus. J Neurovirol 2000;6 (suppl 2):76-79.

40 Jacobson DL, Gange SJ, Rose NR, Graham N Epidemiology and estimated population burden of selected autoimmune diseases in the United States. Clin Immunol Immunopathol 1997;84:223-243. 